EPJ Web of Conferences 61, 04016 (2013)

DOI: $10.1051 /$ epjconf/ 20136104016

(C) Owned by the authors, published by EDP Sciences, 2013

\title{
Broadband variability of TeV BL Lac objects
}

\author{
S. Kaufmann ${ }^{1}$,a , S. Wagner ${ }^{1}$, and O. Tibolla ${ }^{2}$ \\ ${ }^{1}$ Landessternwarte, ZAH, Universität Heidelberg, Germany \\ ${ }^{2}$ ITPA, Universität Würzburg, Germany
}

\begin{abstract}
BL Lac objects provide a direct view on the high energetic jet and offer the possibility to study the emission processes and the particle acceleration in the jet because of their broad emission range over the whole electromagnetic spectrum up to $\mathrm{TeV}$ energies. The variability information gives a direct estimation of the maximum size of the emission region. Under the assumption of the synchrotron Self-Compton model, which is a good description of the spectral energy distribution of BL Lac objects, correlated variability is expected in the different wavebands. The broadband variability is presented for the TeV BL Lac objects PKS 2005-489, RGB J0152+017 and 1ES 0229+200.
\end{abstract}

\section{Introduction}

The emitted radiation of active galactic nuclei (AGN) covers several decades of frequencies. Some AGN can be detected at highest energies, in the $\mathrm{GeV}$ and $\mathrm{TeV} \gamma$-ray regime. Comparing the sky survey catalog at $\mathrm{GeV}$ energies of the Fermi-LAT satellite with an AGN sample, only $\sim 1 \%$ of the AGN are $\gamma$-ray loud AGN (of which $30 \%$ of $\gamma$-ray loud BL Lac objects). While the first generation of Cherenkov telescopes detected only some very bright $\mathrm{TeV}$ sources, the new generation of Imaging Atmospheric Cherenkov Telescopes allowed us to increase the number of $\mathrm{TeV}$ sources to more than 100 with around $30-40 \%$ of AGN. The majority of AGN detected at $\mathrm{TeV}$ energies belong to the class of BL Lac objects.

BL Lac objects are known to be very variable in all detected wavebands. Therefore, simultaneous observations over a broad range, preferably over the whole range of emission, is needed in order to study the underlying emission processes. Due to the variability, every set of simultaneous observations give only a short inside in the actual state of the source.

The spectral energy distribution (SED) of high frequency peaked BL Lac objects are generally characterized by two peaks which are located in the UV-X-ray and the GeV-TeV band, respectively. These are commonly interpreted in terms of leptonic models (e.g. [1]) as synchrotron and inverse Compton (IC) emission from a population of relativistic electrons upscattering their self-produced synchrotron photons (synchrotron self-Compton (SSC) models), e.g. [2], [3]. Under the assumption of such synchrotron Self-Compton model, correlated variability is expected in the different wavebands.

\footnotetext{
a e-mail: S.Kaufmann@1sw.uni-heidelberg.de
}

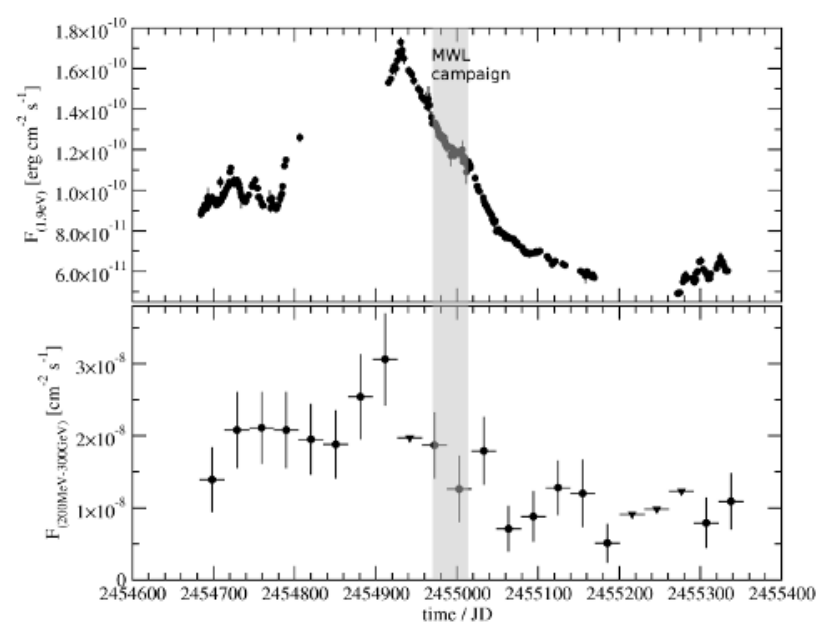

Figure 1. Long term light curves over 22 months of the optical emission by ATOM (upper panel) and the HE $\gamma$-ray emission (lower panel) by Fermi-LAT [6]. The triangles represent upper limits. The grey band indicates the time of the multi-wavelength campaign in 2009.

The broadband variability for the TeV BL Lac objects PKS 2005-489, RGB J0152+017 and 1ES 0229+200 is presented in the following sections.

\section{The BL Lac object with a soft TeV spectrum: PKS 2005-489}

PKS 2005-489 ( $\mathrm{z}=0.071,[4])$ is one of the brightest BL Lac object in the southern hemisphere. TeV $\gamma$-ray emission was first detected with H.E.S.S. [5] and its TeV spectrum is one of the softest among the TeV BL Lac objects.

In 2009, a multi-wavelength campaign with simultaneous observations in the optical, UV, X-ray, GeV and TeV 


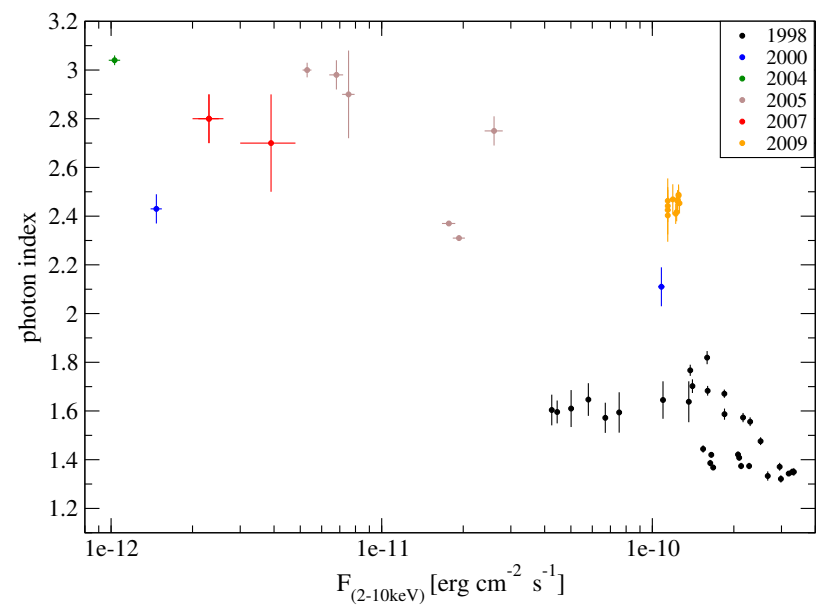

Figure 2. Photon index of a power law describing the X-ray spectra versus the $\mathrm{X}$-ray flux in the energy range $2-10 \mathrm{keV}$

$\gamma$-rays have been performed [7],[6]. Long term monitoring of the $\mathrm{GeV} \gamma$-ray and optical emission revealed variation of the optical emission (Fig. 1). The Fermi-LAT curve shows a variation in the monthly binning with an amplitude similar to the optical variation. Due to the faintness of the source, a monthly binning was used.

The long term variability of PKS 2005-489 has been studied taken into account previous X-ray observations since 1998. The X-ray flux in the energy range $2-10$ $\mathrm{keV}$ varied strongly over two orders of magnitude over 21 years. At the time of the multi wavelength campaign in 2009, PKS 2005-489 was in a very high flux state comparable to the historical maximum of 1998. In addition, the X-ray observations over the last 21 years show a clear trend that the spectra become more soft (higher photon index) with decreasing X-ray flux (see Fig. 2).

During the time of the campaign in 2009, correlated variation is detected in the X-ray and optical emission which cannot be proven on the yearly time scale since the monitoring in X-rays is not as dense as for the optical emission. In 2004 and 2009 simultaneous observations have been obtained in the $\mathrm{X}$-ray and $\mathrm{TeV} \gamma$-ray range (see Fig. 3).

From the SSC model, correlated variations in $\mathrm{GeV} \gamma$ ray and optical emission is expected and was measured. Similar correlation would be also expected for the $\mathrm{TeV} \gamma$ ray and $\mathrm{X}$-ray emission. Instead, Fig. 3 shows that the $\mathrm{X}$ ray flux varied by a factor of 100 while the $\mathrm{TeV} \gamma$-ray flux changed only by a factor of 2 in the simultaneous obtained spectra in 2004 and 2009

\section{The BL Lac object with a low synchrotron peak frequency: RGB J0152+017}

RGB J0152+017 ( $\mathrm{z}=0.08,[8])$ was observed in the optical, UV and X-ray range at the time of its $\mathrm{TeV}$ detection [9] and afterwards [10]. Intra-day-variability with a time scale of $\sim 1 \mathrm{~h}$ show that the emission volume is very

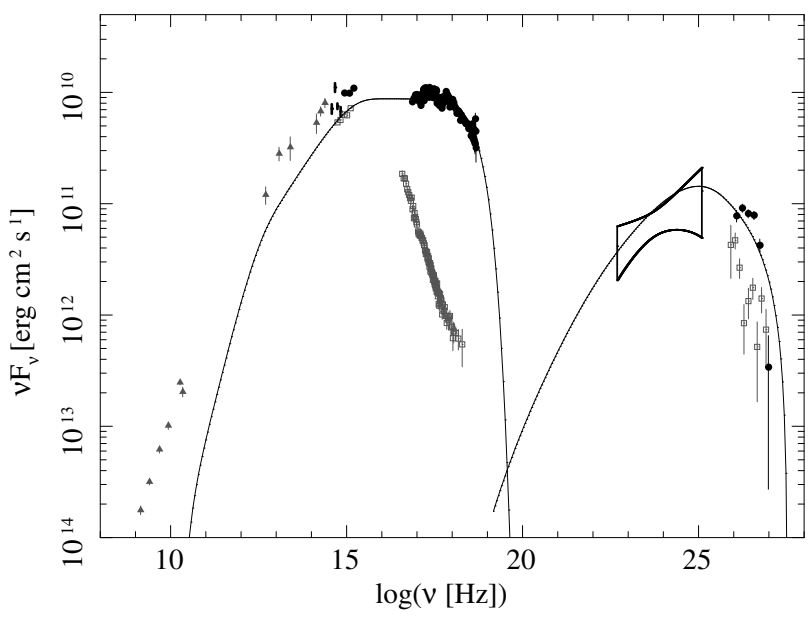

Figure 3. SED of PKS 2005-489 with simultaneous observations of 2009 in black [6]. The spectra are corrected for extinction and absorption effects. UV, X-ray and $\mathrm{TeV} \gamma$-ray data of 2004 are shown as open symbols and historical radio to IR data are shown in grey.

compact. The different variability pattern in UV and Xrays suggest a change of the synchrotron emission. In Fig. 4 strict simultaneous observations in UV and X-ray are shown together with a log-parabolic model to illustrate the change of the synchrotron peak energy over the time of one month. In comparison to other TeV BL Lac objects [11], the synchrotron emission of RGB J0152+017 peaks at very low energy, while it peaks in the $\mathrm{X}$-ray range for most others.

\section{The extreme BL Lac object 1ES $0229+200$}

A multi-wavelength campaign on 1ES $0229+200$ ( $\mathrm{z}=0.14$, [12]) was performed in 2009 with very good spectral coverage of the synchrotron emission [13]. As can be seen in Fig. 5, 1ES 0229+200 is an extreme blazar with synchrotron emission up to $100 \mathrm{keV}$. The parameters of the electron distribution and synchrotron emission are well constrained by the spectra. The optical and UV emission, corrected for the strong influence of the host galaxy and the extinction show a clear cut-off. A high minimum Lorentz factor is needed to explain the cut-off in the optical regime [13]. The optical emission is rather constant and slight variations of the flux in the X-ray regime have been detected over the time scale of years. This indicates a slight change of the makroskopic parameters, e.g. magnetic field, radius, Doppler factor, describing the emission volume.

The hard X-ray spectrum up to $15 \mathrm{keV}$ together with the long-term spectrum by Swift/BAT illustrates that the synchrotron peak is extended up to $\sim 100 \mathrm{keV}$ without any significant cut-off in the X-ray spectrum. 1ES $0229+200$ is defined as a high-frequency peaked BL Lac object, and the measured synchrotron emission peaks at higher frequencies $(>100 \mathrm{keV})$ than usual for high-frequency peaked BL 


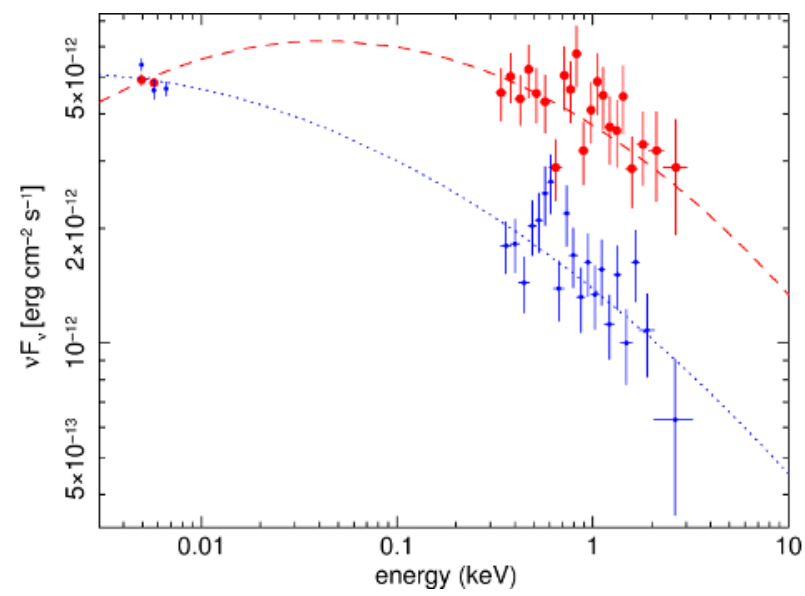

Figure 4. Simultaneous UV and X-ray spectra (corrected for Galactic absorption) shown with the best fitting log-parabolic model of November 15 (red) and December 30, 2007 (blue).

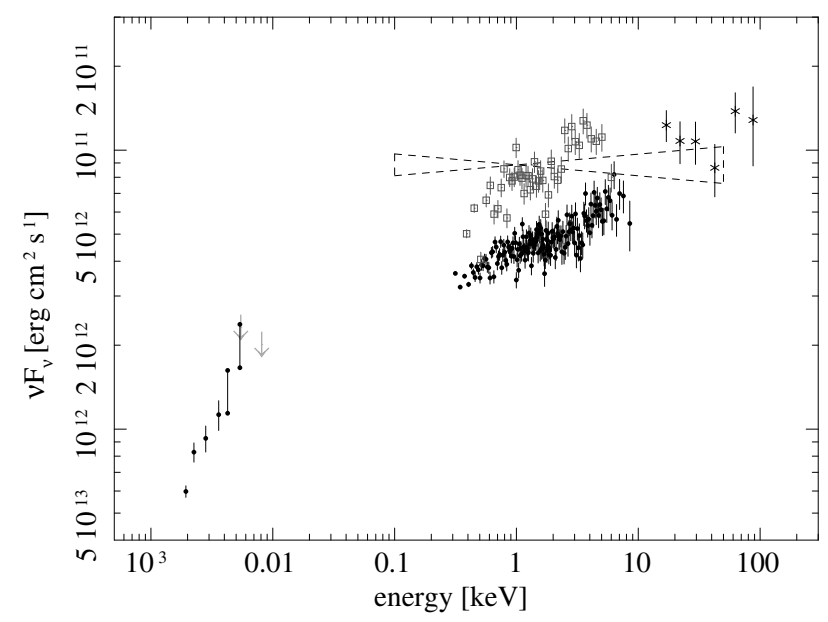

Figure 5. Synchrotron emission of 1ES 0229+200 with simultaneous observations by ATOM and XMM-Newton of August 21, 2009 (black dots) [13]. The emission is corrected by absorption effects and host galaxy influence. Grey open squares represent the highest flux in 2009 by Swift and the butterfly represents the BeppoSAX spectrum of 2001.

Lac objects and belongs therefore to the class of extreme blazars.

\section{Conclusions}

Correlated variation is expected for SSC models and has been found in several TeV BL Lac objects. PKS 2005489 shows similar variation in the optical and $\mathrm{GeV} \gamma$-ray emission. Instead in the X-ray and $\mathrm{TeV} \gamma$-ray emission of
2004 and 2009, strong variation in the X-ray emission exist while only marginal changes in the $\mathrm{TeV} \gamma$-ray emission appear, which is difficult to explain with current emission models.

Strict simultaneous observations in the UV and X-ray range in RGB J0152+017 show a clear shift of the synchrotron emission to very low energies within one month. This represents a direct change of the electron distribution.

The synchrotron emission spectra in the extreme blazar 1ES $0229+200$ show slight flux changes in the Xray emission over years, while the optical emission (dominated by the host galaxy) does not vary.

Broadband variability and correlation studies are being performed for the full sample of $\mathrm{TeV}$ BL Lac objects. Monitoring observations in the synchrotron range exist, while observations in the $\mathrm{TeV} \gamma$-ray range cannot be performed as monitoring due to the long exposure times needed. Therefore every observation gives just a short insight in the emission processes of the highly relativistic jet. The study of the synchrotron emission can give information about the changes of the underlying electron distribution over longer time period.

\section{Acknowledgements}

The authors acknowledge the execution and availability of the XMM-Newton, RXTE and Swift observations and the use of the public HEASARC software packages. S.K. and S.W. acknowledge support from the BMBF through grant DLR 50OR0906.

\section{References}

[1] Marscher, A. P. \& Gear, W. K., ApJ, 298, 114 (1985)

[2] Jones, T.W. et al., ApJ, 188, 353 (1974)

[3] Maraschi, L. et al., ApJL, 397, 5 (1992)

[4] Falomo, R. et al., ApJL, 318, 39 (1987)

[5] Aharonian, F. et al. (H.E.S.S. Collaboration), A\&A, 436, 17 (2005)

[6] H.E.S.S. and Fermi-LAT Collaboration, A\&A, 533, 110 (2011)

[7] Kaufmann, S. et al. for the H.E.S.S. and FermiLAT Collaboration, Fermi Symposium 2009, arXiv:0912.3741 (2009)

[8] Laurent-Mühleisen, S.A., et al., ApJ, 525, 127 (1999)

[9] Aharonian, F. et al. (H.E.S.S. Collaboration), A\&A, 481, 103 (2008)

[10] Kaufmann, S. et al, proceeding of 31st ICRC 2009

[11] Tramacere, A. et al., A\&A, 467, 501 (2007)

[12] Schachter, J.F. et al., ApJ, 412, 541 (1993)

[13] Kaufmann, S. et al, A\&A, 534, 130 (2011) 